\title{
Dynamical corrections to the anomalous holographic soft-wall model: the pomeron and the odderon
}

\author{
Eduardo Folco Capossoli ${ }^{1,2, a}$, Danning $\mathbf{L i}^{3, \mathrm{~b}}$, Henrique Boschi-Filho ${ }^{1, \mathrm{c}}$ \\ ${ }^{1}$ Instituto de Física, Universidade Federal do Rio de Janeiro, Rio de Janeiro, RJ 21.941-972, Brazil \\ ${ }^{2}$ Departamento de Física, Colégio Pedro II, Rio de Janeiro, RJ 20.921-903, Brazil \\ ${ }^{3}$ Institute of Theoretical Physics, Chinese Academy of Science (ITP, CAS), 100190 Beijing, China
}

Received: 8 April 2016 / Accepted: 30 May 2016 / Published online: 11 June 2016

(C) The Author(s) 2016. This article is published with open access at Springerlink.com

\begin{abstract}
In this work we use the holographic soft-wall AdS/QCD model with anomalous dimension contributions coming from two different QCD beta functions to calculate the masses of higher spin glueball states for both even and odd spins and their Regge trajectories, related to the pomeron and the odderon, respectively. We further investigate this model taking into account dynamical corrections due to a dilaton potential consistent with the Einstein equations in five dimensions. The results found in this work for the Regge trajectories within the anomalous soft-wall model with dynamical corrections are consistent with those present in the literature.
\end{abstract}

\section{Introduction}

Quantum chromodynamics (QCD) is a non-Abelian YangMills gauge theory that correctly describes the strong interactions. Its well-known Lagrangian is given by

$\mathcal{L}_{\mathrm{QCD}}=\bar{\psi}(\not D-m) \psi-\frac{1}{4} F_{\mu \nu}^{a} F_{a}^{\mu \nu}$,

where $\psi(x)$ represents the quark fields in the fundamental representation of the SU(3) gauge group and $F_{\mu \nu}^{a}$ is the gluon field strength tensor. This tensor can be decomposed in a such way that

$F_{\mu \nu}^{a}=\partial_{\mu} \mathcal{A}_{\nu}^{a}-\partial_{\nu} \mathcal{A}_{\mu}^{a}+g_{Y M} f^{a b c} \mathcal{A}_{\mu}^{b} \mathcal{A}_{\nu}^{c}$,

where $\mathcal{A}_{v}^{a}$ are the gluon fields, with $a=1, \ldots, 8, f^{a b c}$ are the structure constants of SU(3) group and $g_{\mathrm{YM}}$ is the coupling constant of Yang-Mills (strong) interactions.

Gluons do not carry electric charges, but they have colour charge. Due to this fact, they couple to each other, as implied

\footnotetext{
a e-mail: educapossoli@if.ufrj.br

b e-mail: lidn@itp.ac.cn

c e-mail: boschi@if.ufrj.br
}

by Eqs. (1) and (2). The bound states of gluons predicted by $\mathrm{QCD}$, but not detected so far, are called glueballs. Glueball states are characterised by $J^{P C}$, where $J$ is the total angular momentum, and $P$ and $C$ are the $P$-parity (spatial inversion) and the $C$-parity (charge conjugation) eigenvalues, respectively.

Quantum chromodynamics has many interesting characteristics, among them asymptotic freedom and confinement. The first one means that in the ultraviolet limit, i.e., for high energies or short distances, quarks and gluons practically do not interact with each other. In this case, one has a weak coupling regime and QCD can be treated perturbatively. On the other hand, confinement means that in the infrared limit, i.e., for low energies or large distances, quarks and gluons are bounded to each other strongly, in a strong coupling regime, and therefore, inaccessible to the perturbative approach. Calculations involving bound states as the glueball masses, and consequently, their related Regge trajectories, are features of the non-perturbative regime. Regge trajectories correspond to well-known approximate linear relations between total angular momenta $(J)$ and the square of the masses $(m)$, such that

$J\left(m^{2}\right) \approx \alpha_{0}+\alpha^{\prime} m^{2}$,

with $\alpha_{0}$ and $\alpha^{\prime}$ constants.

In this paper we will calculate the masses for even and odd spin glueball states and from these masses one gets the Regge trajectories for the pomeron and odderon, respectively. As mentioned before those glueball states were not detected experimentally so far. Due to this, the glueball state masses come from some particular models. In the case of the pomeron, related to even spin glueball state masses, there are some models to describe it, such as the soft pomeron [1], given by

$J\left(m^{2}\right) \approx 1.08+0.25 \mathrm{~m}^{2}$, 
where the masses throughout this work are expressed in $\mathrm{GeV}$. There are also other models like the triple pole pomeron [2], the hard pomeron [3], the BFKL pomeron [3] etc. All of these models provide trajectories pretty close to Eq. (4), so we are going to compare our results obtained for the Regge trajectory of the pomeron with this one.

In the case of odderon, related to odd spin glueball states, there are also many models to describe it, such as the isotropic lattice [4], anisotropic lattice [5], relativistic many body [6], given by

$J\left(m^{2}\right) \approx-0.88+0.23 \mathrm{~m}^{2}$,

the non-relativistic constituent [6] given by

$J\left(m^{2}\right) \approx 0.25+0.18 \mathrm{~m}^{2}$,

etc. We are going to compare our results obtained for the Regge trajectory of the odderon, with these two trajectories.

The AdS/CFT or Anti de Sitter/conformal field theory correspondence [7-11] arises as a powerful tool to tackle non-perturbative Yang-Mills theories. The AdS/CFT correspondence relates a conformal Yang-Mills theory with the symmetry group $\mathrm{SU}(N)$ for very large $N$ and extended supersymmetry $(\mathcal{N}=4)$ with a I I $B$ superstring theory in a curved space, known as anti de Sitter space, or $\operatorname{AdS}_{5} \times S^{5}$. At low energies string theory is represented by an effective supergravity theory. Due to this, the AdS/CFT correspondence is also known as a gauge/gravity duality.

After a suitable breaking of the conformal symmetry one can build phenomenological models that describe (large $N$ ) QCD approximately. These models are known as AdS/QCD models.

In order to deal with conformal symmetry breaking the works [12-15] have made some important progress. In these works there emerged the idea of the hard-wall model. This idea means that a hard cutoff is introduced at a certain value $z_{\text {max }}$ of the holographic coordinate $z$ and the space considered is a slice of $\mathrm{AdS}_{5}$ space in the region $0 \leq z \leq z_{\max }$, with some appropriate boundary conditions.

Another holographic approach to break the conformal invariance in the boundary field theory, and make it an effective theory for large $N$ QCD is called the soft-wall model. This model introduces in the action a decreasing exponential factor of the dilatonic field that represents a soft IR cutoff. The soft-wall model (SW) was proposed in [16] to study vector mesons, and subsequently extended to glueballs [17] and to other mesons and baryons [18]. It was shown in Ref. [19] that the soft-wall model does not give the expected masses for the scalar glueball states (and its radial excitations) and higher spin glueball states (with even and odd spins).

In Refs. [20-22] was introduced the idea of using QCD beta functions to get an interesting UV behaviour for the soft-wall model modified by convenient superpotentials for the dilaton field. Then in Ref. [23] a simpler modification was proposed of the SW model, taking into account anomalous dimensions, also related to QCD beta functions to obtain the scalar glueball spectra and its (spin 0) radial excitations. The resulting masses found for some choices of beta functions are in agreement with those presented in the literature.

In this work, our main objective is to extend the previous studies done in Ref. [23] to investigate higher, even and odd, spin glueball states, and obtain the Regge trajectories related to the pomeron and to the odderon, taking into account the anomalous dimensions from some QCD beta functions with dynamical corrections, i.e., considering that the dilatonic field became dynamical satisfying the Einstein equations in five dimensions. Actually, we first consider the anomalous contributions to the original soft-wall model without dynamical corrections for higher even and odd spins, but the results are not good compared with the pomeron and odderon Regge trajectories. Then we consider the dynamical case where the results obtained are good.

This work is organised as follows: In Sect. 2 we provide a quick review of the original soft-wall model (SW) and the modifications taking into account the anomalous dimensions from QCD beta functions that we call the anomalous softwall model (ASW) (that is, the soft-wall with anomalous contributions as proposed in Ref. [23]). Then we introduce even and odd higher spin state operators and calculate, from the mass results, the Regge trajectories related to the pomeron and to the odderon in two different cases: the beta function with a linear asymptotic behaviour and the beta function with an IR fixed point at finite coupling. At this point, we show that the ASW model needs some corrections to provide compatible Regge trajectories related to the pomeron and to the odderon. In Sect. 3 we impose dynamical corrections coming from the dilaton field in the ASW model to achieve the Regge trajectories related to the pomeron and to the odderon. In particular, for the beta function with an IR fixed point at finite coupling, the Regge trajectories obtained for both even and odd spin glueball states, related to the pomeron and the odderon, respectively, are in agreement with those found in the literature. In Sect. 4 we make some comments and summarise our results.

\section{The anomalous soft-wall model}

Let us start this section performing a quick review of the SW model. Then we proceed to discuss the modifications due to the anomalous dimensions of the ASW model, introduce higher spin states and obtain the glueball masses and Regge trajectories.

The soft-wall model for scalar fields can be defined by the action $[17,23]$ 
$S=\frac{1}{\kappa} \int \mathrm{d}^{5} x \sqrt{-g} e^{-\Phi(z)}\left[g^{M N} \partial_{M} X \partial_{N} X+M_{5}^{2} X^{2}\right]$

where $\Phi(z)=k z^{2}$ is the dilatonic field, $M_{5}$ is the fivedimensional mass of the scalar field $X=X(z, x)$ and $g$ is the determinant of the metric tensor of the five-dimensional AdS space described by

$\mathrm{d} s^{2}=g_{M N} \mathrm{~d} x^{M} \mathrm{~d} x^{N}=\frac{R^{2}}{z^{2}}\left(\mathrm{~d} z^{2}+\eta_{\mu \nu} \mathrm{d} x^{\mu} \mathrm{d} x^{\nu}\right)$,

with $M, N=0,1,2,3,4 ; \mu, v=0,1,2,3$, and $\eta_{\mu \nu}=$ diag $(-1,1,1,1)$ is the metric of the four-dimensional Minkowski space. The constant $R$ is called the radius of the $\mathrm{AdS}_{5}$ space.

In the SW model, to find the masses of glueball states one has to solve a "Schrödinger-like" equation,

$$
\begin{aligned}
& -\psi^{\prime \prime}(z)+\left[k^{2} z^{2}+\frac{15}{4 z^{2}}+2 k+\left(\frac{R}{z}\right)^{2} M_{5}^{2}\right] \psi(z) \\
& =\left(-q^{2}\right) \psi(z),
\end{aligned}
$$

corresponding to the equation of motion for the field $X$, as shown in Refs. [16,17]. The glueball masses $m_{n}$, where $m_{n}^{2} \equiv$ $-q^{2}$, can be obtained from normalisable solutions of Eq. (9):

$m_{n}^{2}=\left[4 n+4+2 \sqrt{4+R^{2} M_{5}^{2}}\right] k$,

where $n=0,1,2, \ldots$ denotes the radial (spin 0 ) modes.

From the AdS/CFT dictionary one can show how to relate masses of supergravity fields in the $\mathrm{AdS}_{5} \times S^{5}$ space (here and in the following we disregard the $S^{5}$ space, since the fields are assumed to be independent of it) and scaling dimensions of the local gauge-invariant dual operator in the Super Yang-Mills theory (SYM). For instance, the classical (nonanomalous) conformal dimension $\Delta_{\text {class. }}$ of a super YangMills scalar operator is given by

$\Delta_{\text {class. }}=2+\sqrt{4+R^{2} M_{5}^{2}}$.

Therefore, one can write

$R^{2} M_{5}^{2}=\Delta_{\text {class. }}\left(\Delta_{\text {class. }}-4\right)$.

For a pure SYM theory defined on the boundary of the $\mathrm{AdS}_{5}$ space, the scalar glueball state $0^{++}$is represented by the operator $\mathcal{O}_{4}$, given by

$\mathcal{O}_{4}=\operatorname{Tr}\left(F^{2}\right)=\operatorname{Tr}\left(F^{\mu \nu} F_{\mu \nu}\right)$.

The lightest scalar glueball $0^{++}$is dual to the fields with zero mass $\left(M_{5}^{2}=0\right)$ in the $\mathrm{AdS}_{5}$ space. Then one can see, using (12), that the operator $\mathcal{O}_{4}$ has conformal dimension $\Delta_{\text {class. }}=4$.
The SYM is a conformal theory, so the beta function vanishes and the conformal dimensions have no anomalous contributions, therefore they keep only their classical dimension.

On the other side, for a QCD scalar glueball operator, its full dimension can be computed from the trace anomaly of the energy-momentum tensor [24,25]:

$T_{\mu}^{\mu}=\frac{\beta(\alpha)}{16 \pi \alpha^{2}} \operatorname{Tr} F^{2}+$ fermionic terms

where the beta function is defined by

$\beta(\alpha(\mu)) \equiv \frac{\mathrm{d} \alpha(\mu)}{\mathrm{d} \ln (\mu)}$

and $\mu$ is a renormalisation scale, $\alpha \equiv g_{\mathrm{YM}}^{2} / 4 \pi$ and $g_{\mathrm{YM}}$ is the Yang-Mills coupling constant. The fermionic part in (14) can be disregarded because only the operator $\operatorname{Tr} F^{2}$ is relevant for our purposes. Moreover, the scaling behaviour for a generic operator is given by

$\Delta_{\mathcal{O}}=-\frac{\mathrm{d} \mathcal{O}}{\mathrm{d} \ln \mu}$.

The full dimension $\Delta_{\mathcal{O}}$ also can be seen as a sum of the classical dimension $\Delta_{\text {class. }}$ and the anomalous dimension $\gamma(\mu)$, so that

$\Delta_{\mathcal{O}}=\Delta_{\text {class. }}+\gamma(\mu)$

For the scalar glueball operator, one can insert Eq. (14), without fermionic part, in (16), obtaining

$\Delta_{T_{\mu}^{\mu}}\left(\frac{\beta(\alpha)}{8 \pi \alpha^{2}} \operatorname{Tr} F^{2}\right)=-\frac{\mathrm{d}}{\mathrm{d} \ln \mu}\left(\frac{\beta(\alpha)}{8 \pi \alpha^{2}} \operatorname{Tr} F^{2}\right)$,

or

$$
\begin{aligned}
& \Delta_{T_{\mu}^{\mu}}\left(\frac{\beta(\alpha)}{8 \pi \alpha^{2}} \operatorname{Tr} F^{2}\right) \\
& =-\left(\beta^{\prime}(\alpha)-\frac{2}{\alpha} \beta(\alpha)-\Delta_{F^{2}}\right) \frac{\beta(\alpha)}{8 \pi \alpha^{2}} \operatorname{Tr} F^{2},
\end{aligned}
$$

where the prime represents the derivative with respect to $\alpha$.

The trace $T_{\mu}^{\mu}$ scales classically; that means $\Delta_{T_{\mu}^{\mu} \text { (Class.) }}=$ 4 [25]. So, the scalar glueball operator $\operatorname{Tr} F^{2}$ has the full dimension:

$\Delta_{F^{2}}=4+\beta^{\prime}(\alpha)-\frac{2}{\alpha} \beta(\alpha)$.

Using the 't Hooft coupling, $\lambda \equiv N_{C} g_{\mathrm{YM}}^{2}=4 \pi N_{C} \alpha$, one gets

$\Delta_{F^{2}}=4+\beta^{\prime}(\lambda)-\frac{2}{\lambda} \beta(\lambda)$ 
and now the prime represents the derivative with respect to $\lambda$ and the beta function is given by

$\beta(\lambda(\mu)) \equiv \frac{\mathrm{d} \lambda(\mu)}{\mathrm{d} \ln (\mu)}$

In Ref. [23] this approach was used to study the scalar glueball and its radial (spin 0) excitations spectroscopy due to contributions of anomalous dimensions.

In the present work our concern is with even and odd higher spin glueballs and their corresponding Regge trajectories related to the pomeron and to the odderon.

To get the Regge trajectory for even glueball states we will use the method described in [26] to raise the spin, that is, we will insert $J$ symmetrised covariant derivatives in a given operator with spin $S$, such that the total angular momentum is now $S+J$. In the particular case of the operator $\mathcal{O}_{4}=F^{2}$, one gets

$\mathcal{O}_{4+J}=F D_{\{\mu 1 \ldots} D_{\mu J\}} F$

with conformal dimension $\Delta_{\text {class. }}=4+J$ and spin $J$. Reference [27] used this approach within the hard-wall model to calculate the masses of glueball states $0^{++}, 2^{++}, 4^{++}$, etc. and to obtain the Regge trajectory for the pomeron in agreement with those found in the literature.

So, for even spin glueball states using the soft-wall model after the insertion of symmetrised covariant derivatives, and using $\Delta_{\text {class. }}=4+J$ and $\Delta_{\text {class. }}=2+\sqrt{4+R^{2} M_{5}^{2}}$, one has

$R^{2} M_{5}^{2}=J(J+4) ; \quad($ even $J)$.

In a similar way, one can write the full dimension $\Delta_{T_{\mu}^{\mu}}^{\text {even } J}=4+J$, and now Eq. (21) can be written

$\Delta_{F^{2}}^{\text {even } J}=4+J+\beta^{\prime}(\lambda)-\frac{2}{\lambda} \beta(\lambda)$.

Using (12), the full dimension for a glueball state with higher even spin $J$, taking into account the beta function is

$R^{2} M_{5}^{2}=\Delta_{F^{2}}^{\text {even } J}\left(\Delta_{F^{2}}^{\text {even } J}-4\right)$

or explicitly

$$
\begin{aligned}
R^{2} M_{5}^{2}= & {\left[4+J+\beta^{\prime}(\lambda)-\frac{2}{\lambda} \beta(\lambda)\right] } \\
& \times\left[J+\beta^{\prime}(\lambda)-\frac{2}{\lambda} \beta(\lambda)\right] ; \quad(\text { even } J) .
\end{aligned}
$$

On the other hand, for odd spin glueballs, the operator $\mathcal{O}_{6}$ that describes the glueball state $1^{--}$is given by

$\mathcal{O}_{6}=\operatorname{Sym} \operatorname{Tr}\left(\tilde{F}_{\mu \nu} F^{2}\right)$

and after the insertion of symmetrised covariant derivatives one gets

$\mathcal{O}_{6+J}=\operatorname{Sym} T r\left(\tilde{F}_{\mu \nu} F D_{\{\mu 1 \ldots} D_{\mu J\}} F\right)$

with conformal dimension $\Delta_{\text {class. }}=6+J$ and spin $1+J$. Reference [28] used this approach in the hard-wall model to calculate the masses of odd spin glueball states $1^{--}$, $3^{--}, 5^{--}$etc., and the Regge trajectory for the odderon was obtained in agreement with those found in the literature.

For the case of the odd spin glueball states, using $\Delta=$ $J+6$ and $\Delta=2+\sqrt{4+R^{2} M_{5}^{2}}$, one has

$R^{2} M_{5}^{2}=(J+6)(J+2) ; \quad(\operatorname{odd} J)$

In a similar way, one can write the full dimension $\Delta_{T_{\mu}^{\mu}}^{\text {odd } J}=$ $6+J$, and now Eq. (21) can be written

$\Delta_{F^{2}}^{\text {odd } J}=6+J+\beta^{\prime}(\lambda)-\frac{2}{\lambda} \beta(\lambda)$.

Using (12), one can write the full dimension for a glueball state with higher odd spin $J$, taking into account the beta function:

$R^{2} M_{5}^{2}=\Delta_{F^{2}}^{\mathrm{odd} J}\left(\Delta_{F^{2}}^{\mathrm{odd} J}-4\right)$

and explicitly

$$
\begin{aligned}
R^{2} M_{5}^{2}= & {\left[6+J+\beta^{\prime}(\lambda)-\frac{2}{\lambda} \beta(\lambda)\right] } \\
& \times\left[2+J+\beta^{\prime}(\lambda)-\frac{2}{\lambda} \beta(\lambda)\right] ;(\operatorname{odd} J) .
\end{aligned}
$$

Then, replacing Eqs. (27) and (33) in the Schrödingerlike equation (9) one can solve it numerically and get the masses of higher spin glueballs (even and odd) and consequently obtain the Regge trajectories for the pomeron and the odderon, as we will show below.

At this point, we make a brief comment on QCD beta functions. From perturbative QCD it is well known that one can express the beta function through a power series of the coupling where each term comes from a certain loop order. Exceptionally the two first terms do not depend on the renormalisation set up, but other ones, i.e., higher order terms do.

For our purposes, we will consider some effective nonperturbative beta functions that could reproduce the IR behaviour of QCD as one can see in the works [29-31]. 
We will also impose the requirement that the beta functions reproduce the ultraviolet perturbative behaviour analogous to QCD for small $\lambda$ in 1-loop approximation. That is, $\beta(\lambda) \sim-b_{0} \lambda^{2}$, where $b_{0}$ is a universal coefficient of the perturbative QCD beta function at leading order, given by $b_{0}=\frac{1}{8 \pi^{2}}\left(\frac{11}{3}-\frac{2}{9} N_{f}\right)$. For a pure $\mathrm{SU}(3)_{c}$ one has $N_{f}=0$, then $b_{0}=11 / 24 \pi^{2}$.

From the AdS/QCD soft-wall model one can relate the holographic or radial coordinate $z$ of the $\mathrm{AdS}_{5}$ space with $\mu^{-1}$ where $\mu$ is defined as the renormalisation group scale. So, the relation of the beta function and $z$ is then

$\beta(\lambda(\mu))=\mu \frac{\mathrm{d} \lambda(\mu)}{\mathrm{d} \mu} \Rightarrow \beta(\lambda(z))=-z \frac{\mathrm{d} \lambda(z)}{\mathrm{d} z}$,

where the integration constant will be fixed by $\lambda(z) \equiv \lambda_{0}$ at a particular energy scale $z_{0}$.

In the following subsections the "Schrödinger-like" equation (9) will be solved numerically for two cases, namely, the beta function with a linear asymptotic behaviour and the beta function with an IR fixed point at finite coupling. Then we obtain the corresponding Regge trajectories trying to fit the pomeron and the odderon for each beta function.

\subsection{Beta function with a linear IR asymptotic behaviour}

We begin considering the beta function given in $[29,30]$, such that

$\beta(\lambda)=-\frac{b_{0} \lambda^{2}}{1+b_{1} \lambda} ;$ for $b_{0}, b_{1}>0$.

This beta function behaves as $-b_{0} \lambda^{2}$ in the UV and as $-\left(b_{0} / b_{1}\right) \lambda$ in the IR. Solving Eq. (34) for this beta function, one gets exactly

$\lambda(z)=\frac{1}{b_{1} W\left(\frac{\exp ^{\frac{1}{b_{1} \lambda_{0}}}}{b_{1} \lambda_{0}}\left(\frac{z_{0}}{z}\right)^{b_{0} / b_{1}}\right)}$,

where $W(z)$ is the Lambert function.

Substituting Eqs. (35) and (36) in (27) and (33), then solving numerically the Schrödinger-like equation (9) and using some sets of values for $k, b_{1}$ and $\lambda_{0}$, one can get the Regge trajectories for even and odd glueball states, which could be related to the pomeron and to the odderon, respectively.

The values for $k, b_{1}$ and $\lambda_{0}$, and the results for the Regge trajectories are presented in Table 1, where one can see that the Regge trajectories found for both the pomeron and the odderon for the beta function with a linear IR asymptotic behaviour are in disagreement with (4) for the pomeron [1], and in Eqs. (5) and (6) for the odderon [6]. For instance, in the fourth set of Table 1, one finds that the angular coefficients found for the pomeron and for the odderon are, respectively, $0.35 \pm 0.01$ and $0.32 \pm 0.01 \mathrm{GeV}^{-2}$, which values are much higher than the expect values of $0.25 \mathrm{GeV}^{-2}$ for the pomeron, Eq. (4), and 0.23 or $0.18 \mathrm{GeV}^{-2}$ for the odderon, Eqs. (5) and (6). Since for the other sets the values for the angular coefficients are even higher, we conclude that the ASW model with the beta function with linear asymptotic behaviour does not give good results for the Regge trajectories for the pomeron or for the odderon.

\subsection{Beta function with an IR fixed point at finite coupling}

Here, we consider the beta function given in [31]:

$\beta(\lambda)=-b_{0} \lambda^{2}\left[1-\frac{\lambda}{\lambda_{*}}\right] ;$ for $\lambda_{*}>0$.

This beta function has the necessary IR and UV requirements, as previously discussed. This means that for the IR fixed point $\lambda=\lambda_{*}$ this beta function vanishes. Moreover, it reproduces the perturbative $\beta(\lambda) \sim-b_{0} \lambda^{2}$ at 1-loop order in the ultraviolet and behaves as $\beta(\lambda) \sim+\lambda^{3}$ at large coupling. Equation (34) can also be solved exactly for this beta function, so that

$\lambda(z)=\frac{\lambda_{*}}{1+W\left(\left(\frac{z_{0}}{z}\right)^{b_{0} \lambda_{*}}\left(\frac{\lambda_{*}-\lambda_{0}}{\lambda_{0}}\right) \exp ^{\frac{\lambda_{*}-\lambda_{0}}{\lambda_{0}}}\right)}$

where $W(z)$ is again the Lambert function and $\lambda\left(z_{0}\right)=\lambda_{0}$ fixes the integration constant. This equation leads to the expected QCD asymptotic behaviour at short distances when $z$ is close to the boundary $(z \rightarrow 0)$ :

$\lambda(z) \sim-1 /\left(b_{0} \ln z\right)$.

Replacing (37) and (38) in Eqs. (27) and (33), then solving numerically the Schrödinger-like equation (9) and using some sets of values for $k, \lambda_{0}$ and $\lambda_{*}$, one can get Regge trajectories for even and odd glueball states, which could be related to the pomeron and to the odderon, respectively. The results obtained are presented in Table 2 .

From Table 2, one can see that the Regge trajectories found for both the pomeron and the odderon for the beta function with an IR fixed point in the finite coupling case are in disagreement with those found in [1] for the pomeron, and in [6] for the odderon. As in the case of the previous beta function, for instance, the angular coefficients found here are too high when compared with the ones from the pomeron 0.25 , Eq. (4), and the odderon 0.23 or $0.18 \mathrm{GeV}^{-2}$, Eqs. (5) and (6). So, we conclude that the ASW model with the beta function with an IR fixed point at finite coupling does not give reasonable results for the Regge trajectories for the pomeron or the odderon. 
Table 1 Different values of $k, b_{1}$ and $\lambda_{0}$ used in the ASW model for the beta function with a linear IR asymptotic behaviour, Eq. (35), and the results for the Regge trajectories obtained for the pomeron and the odderon. The errors come from a linear fit

\begin{tabular}{llllll}
\hline Set & $k$ & $b_{1} \times 10^{3}$ & $\lambda_{0}$ & Pomeron & Odderon \\
\hline 1 & -0.25 & 1.2 & 19 & $J \approx(-0.3 \pm 0.3)+(0.42 \pm 0.03) \mathrm{m}^{2}$ & $J \approx(-1.8 \pm 0.5)+(0.40 \pm 0.03) \mathrm{m}^{2}$ \\
2 & -0.49 & 1.2 & 19 & $J \approx(-0.3 \pm 0.4)+(0.39 \pm 0.03) \mathrm{m}^{2}$ & $J \approx(-1.3 \pm 0.4)+(0.34 \pm 0.02) \mathrm{m}^{2}$ \\
3 & -0.72 & 1.2 & 19 & $J \approx(-0.6 \pm 0.4)+(0.38 \pm 0.02) \mathrm{m}^{2}$ & $J \approx(-1.6 \pm 0.3)+(0.33 \pm 0.01) \mathrm{m}^{2}$ \\
4 & -1.00 & 1.2 & 19 & $J \approx(-1.0 \pm 0.3)+(0.35 \pm 0.01) \mathrm{m}^{2}$ & $J \approx(-2.2 \pm 0.3)+(0.32 \pm 0.01) \mathrm{m}^{2}$ \\
5 & -1.00 & 1.2 & 16 & $J \approx(-1.7 \pm 0.2)+(0.45 \pm 0.01) \mathrm{m}^{2}$ & $J \approx(-3.2 \pm 0.2)+(0.43 \pm 0.01) \mathrm{m}^{2}$ \\
6 & -1.00 & 1.0 & 16 & $J \approx(-1.7 \pm 0.2)+(0.45 \pm 0.01) \mathrm{m}^{2}$ & $J \approx(-3.2 \pm 0.2)+(0.42 \pm 0.01) \mathrm{m}^{2}$ \\
\hline
\end{tabular}

Table 2 Regge trajectories obtained for both pomeron and odderon from ASW model using the beta function with an IR fixed point at finite coupling, Eq. (37), and $\lambda_{*}=350$. The errors come from the choice of a linear fit

\begin{tabular}{llll}
\hline$k$ & $\lambda_{0}$ & Pomeron & Odderon \\
\hline-0.36 & 18.5 & $J \approx(-0.9 \pm 0.4)+(0.51 \pm 0.03) \mathrm{m}^{2}$ & $J \approx(-3.0 \pm 0.9)+(0.53 \pm 0.06) \mathrm{m}^{2}$ \\
-0.16 & 18.5 & $J \approx(-4 \pm 2)+(1.4 \pm 0.3) \mathrm{m}^{2}$ & $J \approx(-19.7 \pm 0.3)+(3.06 \pm 0.04) \mathrm{m}^{2}$ \\
-0.36 & 10.5 & $J \approx(-1.93 \pm 0.03)+(1.34 \pm 0.01) \mathrm{m}^{2}$ & $J \approx(-3.82 \pm 0.05)+(1.32 \pm 0.01) \mathrm{m}^{2}$ \\
-0.36 & 25.5 & $J \approx(-3 \pm 2)+(0.57 \pm 0.10) \mathrm{m}^{2}$ & $J \approx(-14 \pm 2)+(1.0 \pm 0.1) \mathrm{m}^{2}$ \\
\hline
\end{tabular}

\section{The dynamical corrections to the anomalous soft-wall model}

In this section we will apply dynamical corrections to modify the ASW model to investigate if these corrections can provide Regge trajectories for the pomeron and the odderon compatible with those found in the literature. To do this, let us perform a quick review of the dynamical soft-wall (DSW) model, discussed in [32-34].

The $5 D$ action for the graviton-dilaton coupling in the string frame is given by

$$
\begin{aligned}
S= & \frac{1}{16 \pi G_{5}} \int \mathrm{d}^{5} x \sqrt{-g_{s}} e^{-2 \Phi(z)} \\
& \times\left(R_{S}+4 \partial_{M} \Phi \partial^{M} \Phi-V_{G}^{S}(\Phi)\right)
\end{aligned}
$$

where $G_{5}$ is the Newton constant in five dimensions, $g_{s}$ is the metric tensor in the five-dimensional space, $\Phi=\Phi(z)$ is the dilaton field and $V_{G}$ is the dilatonic potential. All of these parameters are defined in the string frame. The metric $g_{s}$ has the following form:

$$
\begin{aligned}
\mathrm{d} s^{2}= & g_{M N}^{s} \mathrm{~d} x^{M} \mathrm{~d} x^{N}=b_{s}^{2}(z)\left(\mathrm{d} z^{2}+\eta_{\mu \nu} \mathrm{d} x^{\mu} \mathrm{d} x^{\nu}\right) ; \\
& b_{s}(z) \equiv e^{A_{s}(z)}
\end{aligned}
$$

with $M, N=0,1,2,3,4 ; \mu, v=0,1,2,3$, and $\eta_{\mu \nu}=\operatorname{diag}$ $(-1,1,1,1)$ is the metric of the four-dimensional Minkowski space. Performing a Weyl rescaling, from the string frame to the Einstein frame, one can write Eq. (40) as

$$
S=\frac{1}{16 \pi G_{5}} \int \mathrm{d}^{5} x \sqrt{-g_{E}}\left(R_{E}-\frac{4}{3} \partial_{M} \Phi \partial^{M} \Phi-V_{G}^{E}(\Phi)\right),
$$

where

$g_{\mathrm{MN}}^{E}=g_{M N}^{s} e^{-\frac{2}{3} \Phi} ; \quad V_{G}^{E}=e^{\frac{4}{3} \Phi} V_{G}^{s}$.

Varying the action (42), one can obtain the equations of motion, which are given by

$E_{\mathrm{MN}}+\frac{1}{2} g_{\mathrm{MN}}^{E}\left(\frac{4}{3} \partial_{L} \Phi \partial^{L} \Phi+V_{G}^{E}\right)-\frac{4}{3} \partial_{M} \Phi \partial^{M} \Phi=0$

$\frac{8}{3 \sqrt{g_{E}}} \partial_{M}\left(\sqrt{g_{E}} \partial^{M} \Phi\right)-\partial_{\Phi} V_{G}^{E}(\Phi)=0$,

where $E_{\mathrm{MN}}$ is the Einstein tensor.

Using the metric parametrisation given by (41), the equations of motion (44) and (45) can be written

$$
\begin{aligned}
& -A_{E}^{\prime \prime}+A_{E}^{\prime 2}-\frac{4}{9} \Phi^{\prime 2}=0 \\
& \Phi^{\prime \prime}+3 A_{E}^{\prime} \Phi^{\prime}-\frac{3}{8} e^{2 A_{E}} \partial_{\Phi} V_{G}^{E}(\Phi)=0
\end{aligned}
$$

where we defined

$$
b_{E}(z)=b_{S}(z) e^{-\frac{2}{3} \Phi(z)}=e^{A_{E}(z)} ; \quad A_{E}(z)=A_{s}(z)-\frac{2}{3} \Phi(z) .
$$

Solving Eqs. (46) and (47) for the quadratic dilaton background, $\Phi(z)=k z^{2}$, one finds

$$
A_{E}(z)=\log \left(\frac{R}{z}\right)-\log \left({ }_{0} F_{1}\left(\frac{5}{4}, \frac{\Phi^{2}}{9}\right)\right)
$$


and

$V_{G}^{E}(\Phi)=-\frac{12{ }_{0} F_{1}\left(\frac{1}{4}, \frac{\Phi^{2}}{9}\right)^{2}}{R^{2}}+\frac{16_{0} F_{1}\left(\frac{5}{4}, \frac{\Phi^{2}}{9}\right)^{2} \Phi^{2}}{3 R^{2}}$,

where ${ }_{0} F_{1}(a, z)$ is the Kummer confluent hypergeometric function. Using (48) and (49), one can easily see that

$A_{S}(z)=\log \left(\frac{R}{z}\right)+\frac{2}{3} \Phi(z)-\log \left({ }_{0} F_{1}\left(\frac{5}{4}, \frac{\Phi^{2}}{9}\right)\right)$,

which implies that the metric (41) in this dynamical model is no longer AdS, but it is asymptotically AdS in the limit $z \rightarrow 0$.

The $5 D$ action for the scalar glueball in the string frame is given by $[17,18]$

$S=\int \mathrm{d}^{5} x \sqrt{-g_{s}} \frac{1}{2} e^{-\Phi(z)}\left[\partial_{M} \mathcal{G} \partial^{M} \mathcal{G}+M_{5}^{2} \mathcal{G}^{2}\right]$,

and its equations of motion are

$\partial_{M}\left[\sqrt{-g_{s}} e^{-\Phi(z)} g^{\mathrm{MN}} \partial_{N} \mathcal{G}\right]-\sqrt{-g_{s}} e^{-\Phi(z)} M_{5}^{2} \mathcal{G}=0$.

Using the metric (41), one gets

$\partial_{M}\left[e^{3 A_{s}(z)-\Phi(z)} \eta^{\mathrm{MN}} \partial_{N} \mathcal{G}\right]-e^{5 A_{s}(z)-\Phi(z)} M_{5}^{2} \mathcal{G}=0$.

Doing the substitution $B(z)=\Phi(z)-3 A_{s}(z)$, using the ansatz $\mathcal{G}\left(z, x^{\mu}\right)=v(z) e^{i q_{\mu} x^{\mu}}$, and defining $v(z)=$ $\psi(z) e^{\frac{B(z)}{2}}$, one gets

$-\psi^{\prime \prime}(z)+\left[\frac{B^{\prime 2}(z)}{4}-\frac{B^{\prime \prime}(z)}{2}+\left(\frac{R M_{5}}{z}\right)^{2} e^{4 k z^{2} / 3} \mathcal{A}^{-2}\right]$

$\times \psi(z)=-q^{2} \psi(z)$,

where $\mathcal{A}$ is given by ${ }_{0} F_{1}\left(\frac{5}{4}, \frac{\Phi^{2}}{9}\right)$, or explicitly, for the quadratic dilaton $\Phi(z)=k z^{2}$ :

$-\psi^{\prime \prime}(z)+\left[k^{2} z^{2}+\frac{15}{4 z^{2}}-2 k+\left(\frac{R M_{5}}{z}\right)^{2} e^{4 k z^{2} / 3} \mathcal{A}^{-2}\right]$

$$
\times \psi(z)=-q^{2} \psi(z) .
$$

This equation was solved numerically in [34] and the masses found for the scalar glueball and its radial (spin 0 ) excitations are compatible with those obtained by lattice QCD.

To take into account dynamical corrections plus the anomalous dimension effects, one must recall, from Sect. 2, that for even spin glueball states the full dimension is given by Eq. (25), so that

$\Delta_{F^{2}}^{\text {even } J}=4+J+\beta^{\prime}(\lambda)-\frac{2}{\lambda} \beta(\lambda)$.
For the odd spin glueball state the full dimension is given by Eq. (31), then

$\Delta_{F^{2}}^{\text {odd } J}=6+J+\beta^{\prime}(\lambda)-\frac{2}{\lambda} \beta(\lambda)$.

Using once again the relation $R^{2} M_{5}^{2}=\Delta(\Delta-4)$, one gets

$R^{2} M_{5}^{2}=$

$\begin{cases}{\left[4+J+\beta^{\prime}(\lambda)-\frac{2}{\lambda} \beta(\lambda)\right]\left[J+\beta^{\prime}(\lambda)-\frac{2}{\lambda} \beta(\lambda)\right],} & \text { if } \Delta=\Delta_{F^{2}}^{\text {even } J} \\ {\left[6+J+\beta^{\prime}(\lambda)-\frac{2}{\lambda} \beta(\lambda)\right]\left[2+J+\beta^{\prime}(\lambda)-\frac{2}{\lambda} \beta(\lambda)\right],} & \text { if } \Delta=\Delta_{F^{2}}^{\text {od } J} .\end{cases}$

To consider even or odd spin glueball states one can insert the first or the second line of Eq. (59), respectively, into the Schrödinger-like equation obtained from the DSW model, Eq. (56), and solve it numerically. For our purposes, the same two regimes of the previous section will be studied, namely, the beta function with a linear asymptotic behaviour and the beta function with an IR fixed point at finite coupling. The results will be discussed in the following.

\subsection{Beta function with a linear IR asymptotic behaviour}

Using the beta function given by Eq. (35) and replacing it in Eq. (59) one can solve numerically the Schrödinger-like equation for the DSW model (56) for both even and odd glueball states.

For a set of values for $k, b_{1}$ and $\lambda_{0}$, one can get Regge trajectories for even and odd glueball states. The set used together with the results obtained for Regge trajectories for both the pomeron and the odderon are shown in Table 3.

From Table 3, one can see that the Regge trajectories found for both the pomeron and the odderon with the beta function with a linear IR asymptotic behaviour, Eq. (35), are in disagreement with those found in (4) for the pomeron [1], and in Eqs. (5) and (6) for the odderon [6]. The angular coefficients are too high and the intercepts are too low, both for the pomeron and for the odderon. Other sets of parameters give even poorer results.

\subsection{Beta function with an IR fixed point at finite coupling}

Using the beta function given by Eq. (37) and substituting it into Eq. (59), one can solve numerically the Schrödinger-like equation (56) for both even and odd glueball states.

We consider different sets of values for $k, \lambda_{0}$ and $\lambda_{*}$ and get the masses of the glueball states with even and odd spins and the Regge trajectories related to the pomeron and to the odderon, respectively. The sets of parameters used and the results obtained for the glueball state masses with even and odd spins are presented in Table 4 . The results obtained for Regge trajectories are shown in Table 5. 
Table 3 Parameters used in the beta function with a linear IR asymptotic behaviour, Eq. (35), and the corresponding Regge trajectories obtained for both the pomeron and the odderon from the anomalous and dynamical soft-wall model, Eqs. (56) and (59). The errors come from the choice of a linear fit

\begin{tabular}{lllll}
\hline$k$ & $b_{1} \times 10^{3}$ & $\lambda_{0}$ & Pomeron & Odderon \\
\hline-0.25 & 1.2 & 19 & $J \approx(-14 \pm 1)+(4.1 \pm 0.3) \mathrm{m}^{2}$ & $J \approx(-21 \pm 1)+(5.0 \pm 0.3) \mathrm{m}^{2}$
\end{tabular}

Table 4 Masses $(\mathrm{GeV})$ for the glueball states $J^{P C}$ with even and odd $J$ with $P=C= \pm 1$ calculated from the anomalous dynamical soft-wall model, Eqs. (56) and (59), and the beta function with an IR fixed point at finite coupling, (37), using five sets of parameters $k\left(\mathrm{GeV}^{2}\right), \lambda_{0}$ and $\lambda_{*}$ (dimensionless)

\begin{tabular}{|c|c|c|c|c|c|c|c|c|c|c|c|c|c|c|c|}
\hline \multirow[t]{2}{*}{$\overline{\text { Set }}$} & \multicolumn{3}{|c|}{ Parameters } & \multicolumn{12}{|c|}{ Glueball states $J^{P C}$} \\
\hline & $k$ & $\lambda_{0}$ & $\lambda_{*}$ & $0^{++}$ & $2^{++}$ & $4^{++}$ & $6^{++}$ & $8^{++}$ & $10^{++}$ & $1^{--}$ & $3^{--}$ & $5^{--}$ & $7^{--}$ & $9^{--}$ & $11^{--}$ \\
\hline 1 & 0.16 & 18.5 & 350 & 1.69 & 3.28 & 4.76 & 6.23 & 7.67 & 9.12 & 4.02 & 5.50 & 6.95 & 8.40 & 9.84 & 10.00 \\
\hline 2 & 0.09 & 18.5 & 350 & 1.62 & 2.84 & 4.00 & 5.14 & 6.26 & 7.37 & 3.42 & 4.57 & 5.70 & 6.82 & 7.93 & 9.04 \\
\hline 3 & 0.04 & 18.5 & 350 & 1.56 & 2.52 & 3.43 & 4.32 & 5.19 & 6.05 & 2.98 & 3.88 & 4.76 & 5.62 & 6.48 & 7.32 \\
\hline 4 & 0.09 & 10.5 & 350 & 0.79 & 2.13 & 3.28 & 4.39 & 5.48 & 6.57 & 2.72 & 3.84 & 4.94 & 6.03 & 7.11 & 8.19 \\
\hline 5 & 0.09 & 18.5 & 250 & 1.64 & 2.86 & 4.02 & 5.16 & 6.28 & 7.39 & 3.44 & 4.59 & 5.72 & 6.84 & 7.95 & 9.05 \\
\hline
\end{tabular}

Table 5 Regge trajectories obtained for both pomeron and odderon from the anomalous soft-wall model with dynamical corrections, Eqs. (56) and (59), using the beta function with an IR fixed point at finite coupling, (37), for the sets of parameters presented in Table 4. The errors come from the choice of a linear fit

\begin{tabular}{lll}
\hline Set & Pomeron & Odderon \\
\hline 1 & $J \approx(0.6 \pm 0.5)+(0.12 \pm 0.01) \mathrm{m}^{2}$ & $J \approx(-0.1 \pm 0.4)+(0.10 \pm 0.01) \mathrm{m}^{2}$ \\
2 & $J \approx(0.4 \pm 0.5)+(0.19 \pm 0.02) \mathrm{m}^{2}$ & $J \approx(-0.4 \pm 0.4)+(0.15 \pm 0.01) \mathrm{m}^{2}$ \\
3 & $J \approx(0.1 \pm 0.5)+(0.28 \pm 0.02) \mathrm{m}^{2}$ & $J \approx(-0.8 \pm 0.4)+(0.24 \pm 0.02) \mathrm{m}^{2}$ \\
4 & $J \approx(0.9 \pm 0.5)+(0.23 \pm 0.02) \mathrm{m}^{2}$ & $J \approx(0.1 \pm 0.4)+(0.18 \pm 0.01) \mathrm{m}^{2}$ \\
5 & $J \approx(0.4 \pm 0.5)+(0.19 \pm 0.02) \mathrm{m}^{2}$ & $J \approx(-0.4 \pm 0.4)+(0.15 \pm 0.01) \mathrm{m}^{2}$ \\
\hline
\end{tabular}
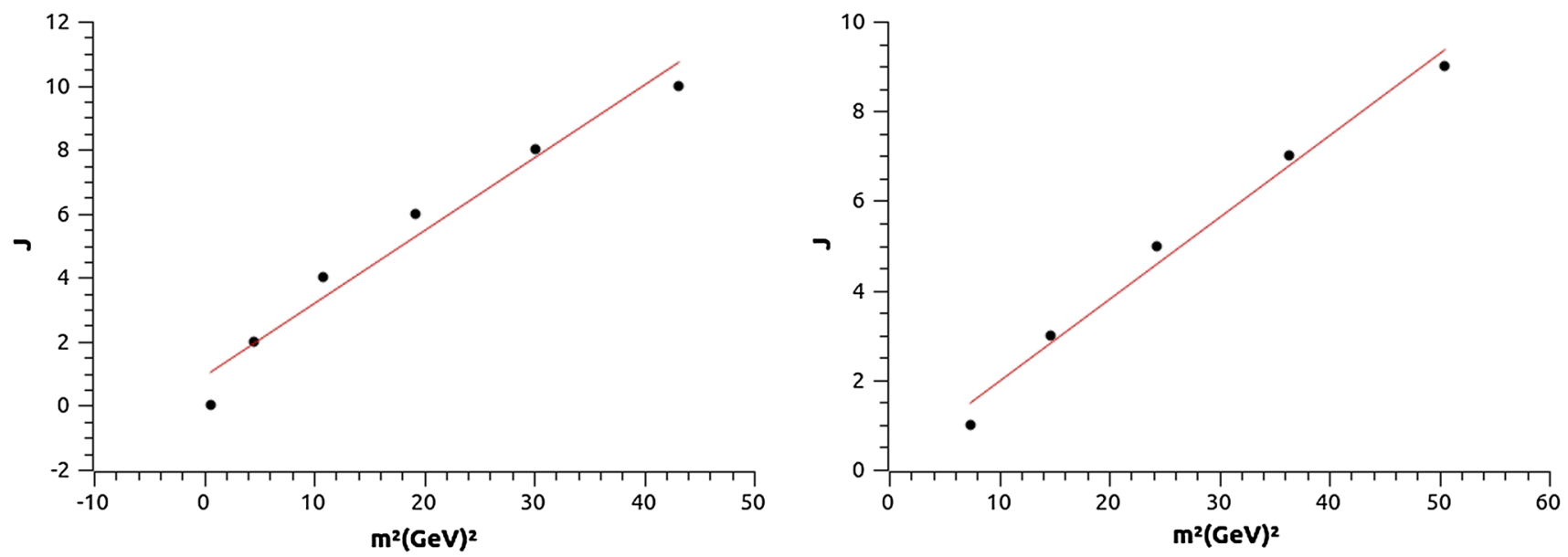

Fig. 1 Approximate Regge trajectories for the pomeron (left) and odderon (right) using data from Table 4 with set number 4 . The pomeron Regge trajectory corresponds to the states $0^{++}, \ldots, 10^{++}$, and the odderon Regge trajectory to the states $1^{--}, \ldots, 9^{--}$

From Table 5, one can see that the Regge trajectories found, using set number 4 presented in Table 4 , for both pomeron and odderon for the beta function with an IR fixed point in the finite coupling regime are in agreement with those found in (4) for the pomeron [1], and with the nonrelativistic constituent model, Eq. (6), for the odderon [6]. In particular, the Regge trajectory related to the pomeron from Table 5 with set number 4 is given by 

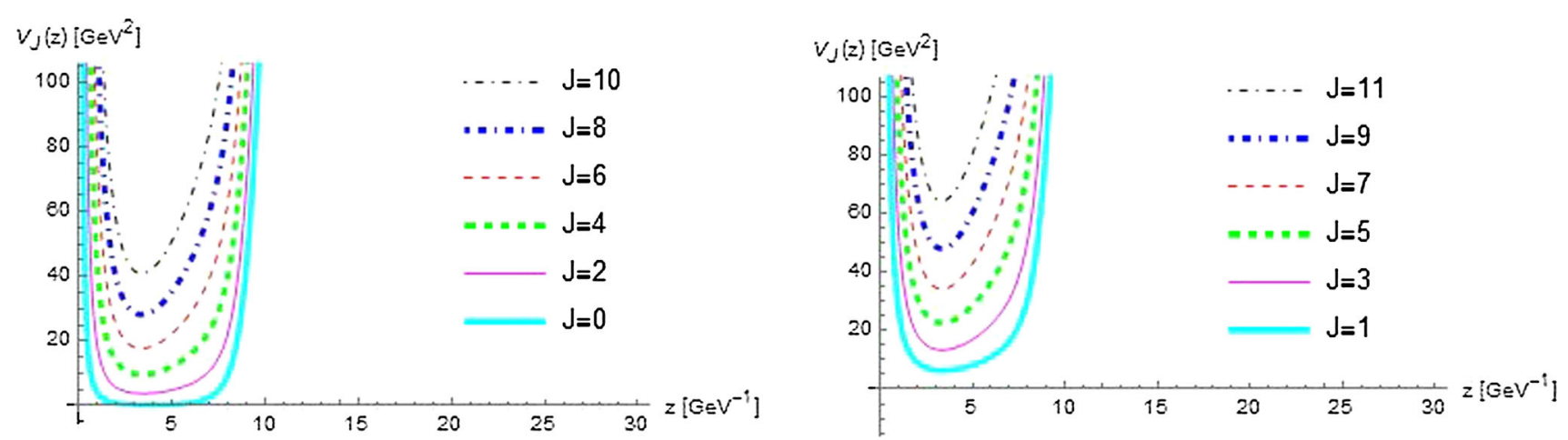

Fig. 2 Plots for the effective potential $V_{J}(z)$ of the dynamical anomalous soft-wall model against the holographic coordinate $z$ for even spins $J=0, \ldots, 10$ (left), and for odd spins $J=1, \ldots, 11$ (right)

$J\left(m^{2}\right) \approx(0.9 \pm 0.5)+(0.23 \pm 0.02) m^{2}$

and is shown in the left panel of Fig. 1. The Regge trajectory related to the odderon from Table 5 with set number 4 , excluding the glueball state $11^{--}$, is given by

$J\left(m^{2}\right) \approx(0.1 \pm 0.4)+(0.18 \pm 0.01) m^{2}$,

which is shown in the right panel of Fig. 1. Here, we excluded the state $11^{--}$because the Regge trajectory corresponding with it was not good when compared with the odderon results, Eqs. (5) and (6). Note that this happens due to the non-linear behaviour of the quadratic masses of the odd spin glueballs, which can be computed by using the values for the masses found in Table 4. Note also that in the original soft-wall model the Regge trajectories are linear, but here in the dynamical version of this model the trajectories are no longer linear. As we are going to show in the next section, this happens because the dynamical corrections produce effective potentials which render this model similar to the hard-wall model, which provides non-linear trajectories. These non-linear behaviours also lead to large uncertainties for the intercepts. As mentioned before, those uncertainties come from the usual linear (least squares) regression fit.

\section{Discussion and conclusions}

In this work we used the anomalous dimension soft-wall model, related to QCD beta functions to obtain high spin glueball masses and the corresponding Regge trajectories. Then we take into account the dynamical corrections caused by the dilaton field, such that the model becomes a solution of Einstein's equations in five dimensions. We take this anomalous and dynamical model to calculate the masses of glueball states with even and odd spins.

Our motivation to consider these dynamical corrections, as was shown in Sect. 2, is due to the fact that although the ASW model worked well for scalar glueball states and its radial (spin 0) excitations [23], this model seems not to work well for higher spin glueball states for both QCD beta functions studied, namely, the beta function with an IR fixed point at finite coupling and the beta function with a linear asymptotic behaviour.

In this work, in particular, for beta function with an IR fixed point at finite coupling, using the fourth set of parameters $k, \lambda_{0}$ and $\lambda_{*}$, shown in Table 4 , the values were found for the masses of the glueball states, both for higher even and odd spins, to be comparable with those found in the literature. The same beta function still provides Regge trajectories, for the pomeron and the odderon, as shown in Eqs. (60) and (61), respectively, in agreement with [1], for the pomeron, and in [6] for the odderon within the non-relativistic constituent model.

This leaves us with a question: why do the soft-wall models with or without anomalous corrections not work for high spin glueballs while the model with dynamical corrections does? To answer this question we plot the effective potentials of the anomalous dynamical soft-wall model for high spins discussed in this work in Fig. 2. The effective potentials are given by

$$
\begin{aligned}
V_{\text {even } J}= & k^{2} z^{2}+\frac{15}{4 z^{2}}+2 k+\frac{1}{z^{2}}\left[4+J+\beta^{\prime}(\lambda)-\frac{2}{\lambda} \beta(\lambda)\right] \\
\times & {\left[J+\beta^{\prime}(\lambda)-\frac{2}{\lambda} \beta(\lambda)\right], } \\
V_{\text {odd } J}= & k^{2} z^{2}+\frac{15}{4 z^{2}}+2 k+\frac{1}{z^{2}}\left[6+J+\beta^{\prime}(\lambda)-\frac{2}{\lambda} \beta(\lambda)\right] \\
\times & {\left[2+J+\beta^{\prime}(\lambda)-\frac{2}{\lambda} \beta(\lambda)\right] . }
\end{aligned}
$$

One can see in Fig. 2 that the effective potentials show an abrupt rise in the IR region (large $z$ ), simulating a wall or in this case a hard-wall. As is well known, the hard-wall model (with Neumann boundary condition) gives good results for even [27] and odd spins [28] related to the pomeron and to 
the odderon, respectively. In fact, the masses found in Table 4 are pretty close to the ones obtained from the hard-wall model with Neumann boundary conditions.

So, we conclude that the dynamical corrections lead to effective potentials that work like a hard-wall at some finite value of the holographic coordinate $z$ implying good results for the glueball masses and Regge trajectories. Similar results have also been recently found for the (non-anomalous) dynamical soft-wall model [35] and for a modified (analytical) soft-wall model [19].

Acknowledgments H.B.-F. is partially supported by CNPq and E.F.C. by CNPq and FAPERJ, Brazilian agencies. D.L. is supported by the China Postdoctoral Science Foundation.

Open Access This article is distributed under the terms of the Creative Commons Attribution 4.0 International License (http://creativecomm ons.org/licenses/by/4.0/), which permits unrestricted use, distribution, and reproduction in any medium, provided you give appropriate credit to the original author(s) and the source, provide a link to the Creative Commons license, and indicate if changes were made. Funded by SCOAP ${ }^{3}$.

\section{References}

1. P.V. Landshoff, Pomerons. arXiv:hep-ph/0108156

2. J.R. Cudell, G. Soyez, Does F(2) need a hard pomeron? Phys. Lett. B 516, 77 (2001). arXiv:hep-ph/0106307

3. E. Levin, An introduction to pomerons. arXiv:hep-ph/9808486

4. H.B. Meyer, M.J. Teper, Glueball Regge trajectories and the pomeron: a Lattice study. Phys. Lett. B 605, 344 (2005). arXiv:hep-ph/0409183

5. Y. Chen et al., Glueball spectrum and matrix elements on anisotropic lattices. Phys. Rev. D 73, 014516 (2006). arXiv:hep-lat/0510074

6. F.J. Llanes-Estrada, P. Bicudo, S.R. Cotanch, Oddballs and a low odderon intercept. Phys. Rev. Lett. 96, 081601 (2006). arXiv:hep-ph/0507205

7. J.M. Maldacena, The Large N limit of superconformal field theories and supergravity. Adv. Theor. Math. Phys. 2, 231 (1998). arXiv:hep-th/9711200

8. S.S. Gubser, I.R. Klebanov, A.M. Polyakov, Gauge theory correlators from noncritical string theory. Phys. Lett. B 428, 105 (1998). arXiv:hep-th/9802109

9. E. Witten, Anti-de Sitter space and holography. Adv. Theor. Math. Phys. 2, 253 (1998). arXiv:hep-th/9802150

10. E. Witten, Anti-de Sitter space, thermal phase transition, and confinement in gauge theories. Adv. Theor. Math. Phys. 2, 505 (1998). arXiv:hep-th/9803131

11. O. Aharony, S.S. Gubser, J.M. Maldacena, H. Ooguri, Y. Oz, Large $\mathrm{N}$ field theories, string theory and gravity. Phys. Rep. 323, 183 (2000). arXiv:hep-th/9905111

12. J. Polchinski, M.J. Strassler, Hard scattering and gauge/string duality. Phys. Rev. Lett. 88, 031601 (2002). arXiv:hep-th/0109174

13. J. Polchinski, M.J. Strassler, Deep inelastic scattering and gauge/string duality. JHEP 0305, 012 (2003). arXiv:hep-th/0209211

14. H. Boschi-Filho, N.R.F. Braga, Gauge/string duality and scalar glueball mass ratios. JHEP 0305, 009 (2003). arXiv:hep-th/0212207
15. H. Boschi-Filho, N.R.F. Braga, QCD/string holographic mapping and glueball mass spectrum. Eur. Phys. J. C 32, 529 (2004). arXiv:hep-th/0209080

16. A. Karch, E. Katz, D.T. Son, M.A. Stephanov, Linear confinement and AdS/QCD. Phys. Rev. D 74, 015005 (2006). arXiv:hep-ph/0602229

17. P. Colangelo, F. De Fazio, F. Jugeau, S. Nicotri, On the light glueball spectrum in a holographic description of QCD. Phys. Lett. B 652, 73 (2007). arXiv:hep-ph/0703316

18. H. Forkel, M. Beyer, T. Frederico, Linear meson and baryon trajectories in AdS/QCD. Int. J. Mod. Phys. E 16, 2794 (2007). arXiv:0705.4115 [hep-ph]

19. E.F. Capossoli, H. Boschi-Filho, Glueball spectra and Regge trajectories from a modified holographic softwall model. Phys. Lett. B 753, 419 (2016). arXiv:1510.03372 [hep-ph]

20. U. Gursoy, E. Kiritsis, Exploring improved holographic theories for QCD: part I. JHEP 0802, 032 (2008). arXiv:0707.1324 [hep-th]

21. U. Gursoy, E. Kiritsis, F. Nitti, Exploring improved holographic theories for QCD: part II. JHEP 0802, 019 (2008). arXiv:0707.1349 [hep-th]

22. U. Gursoy, E. Kiritsis, L. Mazzanti, G. Michalogiorgakis, F. Nitti, Improved holographic QCD. Lect. Notes Phys. 828, 79 (2011). arXiv:1006.5461 [hep-th]

23. H. Boschi-Filho, N.R.F. Braga, F. Jugeau, M.A.C. Torres, Anomalous dimensions and scalar glueball spectroscopy in AdS/QCD. Eur. Phys. J. C 73, 2540 (2013). arXiv:1208.2291 [hep-th]

24. S. Narison, G. Veneziano, QCD tests of $G(1.6)=$ Glueball. Int. J. Mod. Phys. A 4, 2751 (1989)

25. S.S. Gubser, A. Nellore, S.S. Pufu, F.D. Rocha, Thermodynamics and bulk viscosity of approximate black hole duals to finite temperature quantum chromodynamics. Phys. Rev. Lett. 101, 131601 (2008). arXiv:0804.1950 [hep-th]

26. G.F. de Teramond, S.J. Brodsky, Hadronic spectrum of a holographic dual of QCD. Phys. Rev. Lett. 94, 201601 (2005). arXiv:hep-th/0501022

27. H. Boschi-Filho, N.R.F. Braga, H.L. Carrion, Glueball Regge trajectories from gauge/string duality and the Pomeron. Phys. Rev. D 73, 047901 (2006). arXiv:hep-th/0507063

28. E.F. Capossoli, H. Boschi-Filho, Odd spin glueball masses and the Odderon Regge trajectories from the holographic hardwall model. Phys. Rev. D 88(2), 026010 (2013) arXiv:1301.4457 [hep-th]

29. D.F. Zeng, Heavy quark potentials in some renormalization group revised AdS/QCD models. Phys. Rev. D 78, 126006 (2008). arXiv:0805.2733 [hep-th]

30. T.A. Ryttov, F. Sannino, Supersymmetry inspired QCD beta function. Phys. Rev. D 78, 065001 (2008). arXiv:0711.3745 [hep-th]

31. J. Alanen, K. Kajantie, Thermodynamics of a field theory with infrared fixed point from gauge/gravity duality. Phys. Rev. D 81, 046003 (2010). arXiv:0912.4128 [hep-ph]

32. J.P. Shock, F. Wu, Y.L. Wu, Z.F. Xie, AdS/QCD phenomenological models from a back-reacted geometry. JHEP 0703, 064 (2007). arXiv:hep-ph/0611227

33. C.D. White, The Cornell potential from general geometries in AdS/QCD. Phys. Lett. B 652, 79 (2007). arXiv:hep-ph/0701157

34. D. Li, M. Huang, Dynamical holographic QCD model for glueball and light meson spectra. JHEP 1311, 088 (2013). arXiv:1303.6929 [hep-ph]

35. E.F. Capossoli, D. Li, H. Boschi-Filho, Pomeron and Odderon Regge trajectories from a dynamical holographic model. arXiv:1601.05114 [hep-ph] 